\title{
Zur dielektrischen Anisotropie des Naturholzes im großen Frequenzbereich
}

\author{
Kröner, Karl \\ Pungs, Leo \\ Veröffentlicht in: \\ Abhandlungen der Braunschweigischen \\ Wissenschaftlichen Gesellschaft Band 1, 1949, \\ S.101-108
}

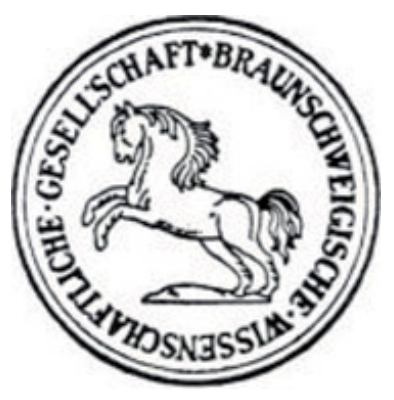

Friedr. Vieweg \& Sohn, Braunschweig 


\title{
Zur dielektrischen Anisotropie des Naturholzes im großen Frequenzbereich
}

\author{
Von Karl Kröner und Leo Pungs
}

Mit 3 Abbildungen

\begin{abstract}
Summary. The dielectrical anisotropy of wood (spruce, beech and oak) in absolutely dry condition is determined by measurements of the dielectrical constant in the frequency range of $3 \cdot 10^{9}$ to $10^{9} \mathrm{c}$. p. s. It is found that the anisotropy is mainly caused by the macroscopical structure of wood as a compositum of cellular substance and air. - An anomalous dispersion of the dielectrical constant is found. The variation of the dispersion caused by different directions of the electrical field is explained likewise by the mixed structure.
\end{abstract}

\section{Allgemeines}

Uber die mechanischen Eigenschaften des Holzes als eines anisotropen Körpers ist ein sehr ausgedehntes Schrifttum vorhanden. Helmholtz') vergleicht ein nicht zu großes Holzstück aus einem regelmäßig gewachsenen Baum mit einem Kristall des rhombischen Systems. Carrington, Hörig und andere haben das elastische Verhalten des Holzes durch einen Vergleich mit einem rhombischen Kristall abgeleitet.

Die dielektrische Anisotropie des Holzes im ganzen Bereich der in der Niederund Hochfrequenztechnik angewandten Schwingungszahlen ist nach der uns z. Z. zugänglichen Literatur kaum behandelt worden. Bei verschiedenen veröffentlichten Meßwerten über die Dielektrizitätskonstante fehlt oft die Angabo der Schnittrichtung. Nun hat wegen der Anwendung der Hochfrequenzenergio zur Erhitzung des Holzes in der Industrie diese Frage auch praktische Bedeutung. Wir haben deshalb über das dielektrische Verhalten des Holzes in bezug auf die DK. und den Verlustfaktor unter der Berücksichtigung der Anisotropie eine Reihe von Untersuchungen im Frequenzbereich von $3 \cdot 10^{2}$ bis $1 \cdot 10^{9} \mathrm{~Hz}$ ausgeführt (Wellenlänge von $1000 \mathrm{~km}$ bis $30 \mathrm{~cm}$ ), über die wir hier als Beitrag zu dieser Frage kurz berichten wollen. Wir beschränken uns auf das Verhalten der DK., während die Behandlung der Verluste an einer anderen Stelle erfolgen wird.

\section{Experimentelle Ergebnisse}

Abb. I stellt einen kleinen Holzwürfel schematisch dar, der aus einem starken Stamm möglichst weit von der Achse ausgeschnitten gedacht ist. , Die den Jahresringen entsprechenden Flächen können dann als annähernd eben angesehen werden. Sie sind durch die feinen Iinien angedeutet. Die gestrichelt gezeichneten Flächen sind die drei Symmetrieebenen, die durch den anatomischen Bau des Holzes gegeben sind. Diesen Ebenen entsprechen als Schnittlinien dio 3 Achsen $h, t, r$. Die Achse $h$ verläuft in Richtung der natürlichen Achse dee Stammes. Fin Schnitt in der zu ihr senkrechten Symmetrieebene $A B$ wird 
als Hirnschnitt bezeichnet. Der Tangentialschnitt, tangential zu den Jahresringen, verläuft parallel zu der Ebene $C D$, senkrecht zur Achse $t$, der Radialschnitt $E F$ senkrecht zu den Jahresringen. Bei der Behandlung der Elastizitätstheorie des Holzes im Vergleich mit einem Kristall werden die drei Achsen denjenigen des rhombischen Systems gleichgesetzt. Selbstverständlich sind die Bedingungen der Symmetrie für das Naturholz nur mit grober Annäherung erfüllt. Der Grund liegt in der verschiedenen Breite der Jahresringe, dem verschiedenen Gefüge des Früh- und Spätholzes usw.

Bei unseren Untersuchungen haben wir kreisförmige Scheiben benutzt, deren Schnittflächen den 3 genannten Symmetrieebenen parallel waren. Die

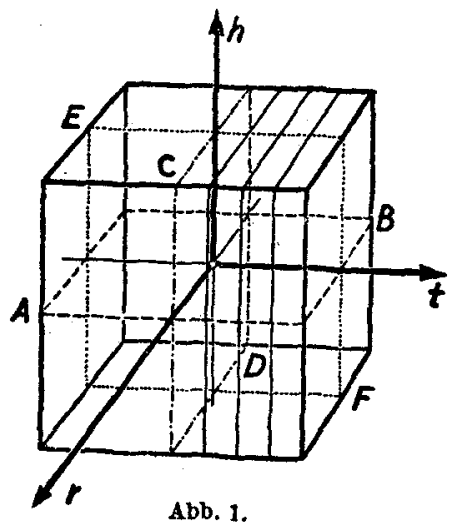

Schema der Symmetrieebenen bet Holz.

Richtungen des elektrischen Feldes entsprachen also den 3 Achsen $h, t$ und $r$. Die Holzproben wurden, um einheitliche Ergebnisse zu erhalten und den Einfluß der Feuchtigkeit auszuschließen, im Darrzustand untersucht. Der Darrzustand wird bekanntlich erreicht, wenn man das $\mathrm{Holz}$ bei $100^{\circ} \mathrm{C}$ im Trockenofen solange erhitzt, bis keine Gewichtsabnahme stattfindet. Die Messungen müssen sofort nach einer $A b$ kühlung im Exsikkator erfolgen, um die Aufnahme der Feuchtigkeit aus der Luft zu verhindern. Die Temperatur war bei den Versuchen durchgehend $20^{\circ} \mathrm{C}$. Der Einfluß der Feuchtigkeit auf das dielektrische Verhalten wird in einer weiteren Arbeit behandelt werden.

Die DK. wurde in bekannter Weise durch Messung der Kapazität bestimmt. Bei Frequenzen von 300 bis $10^{4} \mathrm{~Hz}$ wurde die Brückenmethode, von $10^{4}$ bis $10^{9} \mathrm{~Hz}$ die Resonanzmethode angewandt, und zwar bis $10^{8} \mathrm{~Hz}$ mit einem geschlossenen Schwingungssystem, bis $10^{9} \mathrm{~Hz}$ mit einem Paralleldrahtsystem.

Aus den Ergebnissen sind die Meßwerte für zwei typische Holzarten, und zwar für Fichte als Nadelholz und für Rotbuche als Laubholz, in den Abb. 2 und 3 dargestellt. Die DK. der drei Schnitte sind wie folgt bezeichnet:

$$
\begin{aligned}
& \varepsilon_{h}=\text { Hirnschnitt } \\
& \varepsilon_{t}=\text { Tangentialschnitt }, \\
& \varepsilon_{r}=\text { Radialschnitt. }
\end{aligned}
$$

Die Kurven stellen die Werte in Abbängigkeit von der Frequenz dar, die im logarithmischen Maßstab aufgetragen ist. Wir geben zum Vergleich noch die Werte für Eichenholz für zwei Frequenzen an.

$\begin{array}{cccc}f & \text { Eiche } \\ 3 \cdot 10^{2} & \varepsilon_{h} & \varepsilon_{t} & \varepsilon_{r} \\ 1 \cdot 10^{8} & 2,86 & 2,30 & 2,46 \\ & 2,16 & 1,96 & 1,82\end{array}$

Die Messungen zeigen folgendes:

I. Die DK. hat bei allen untersuchten Hölzern den größten Wert, wesn die Richtung des elektrischen Feldes senkrecht zum Hirnschnitt verläuft, während 
für die beiden anderen Schnitte sich kleinere und voneinander nur wenig verschiedene Werte ergeben. Die kleinste Anisotropie zeigt das Eichenholz. Für die Anisotropie ist die Faserrichtung in erster Linie maßgebend. Der Vergleich mit den mechanischen Eigenschaften zeigt, daß die Feldrichtung für die größte DK. mit der Richtung des größten Elastizitätsmoduls und der größten Zugfestigkeit zusammenfällt. Nur ist die mechanische Anisotropie viel ausgeprägter als die dielektrische.

2. Der Verlauf der DK. in Abhängigkeit von der Frequenz zeigt eine ausgesprochene im optischen Sinne anomale Dispersion. Die DK. fällt mit steigender

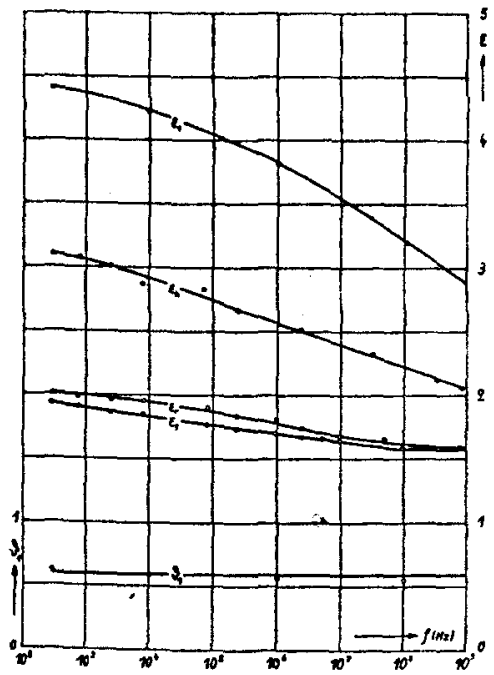

Abb. 2. Dielektrizitatskonstante in verschiedenen Schnittrichtungen von Fichtenholz

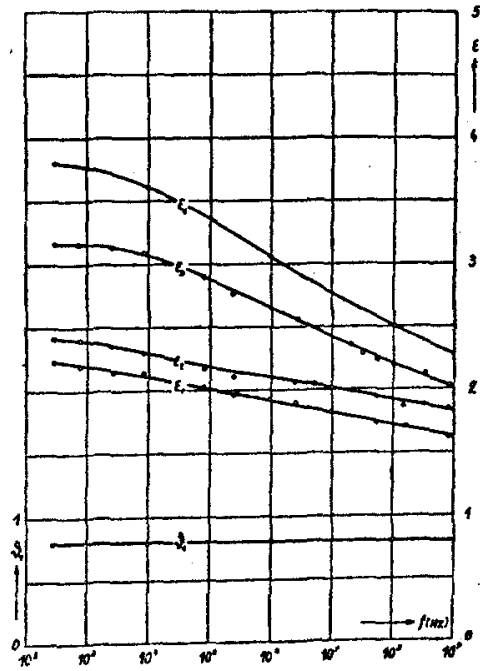

Abb. 3. Dielektrizitatskonstante in verschledenen Schnittrichtungen von- Rotbuchenliola

Frequenz ähnlich wie bei Stoffen mit ausgeprägten Molekulardipolen, jedoch ohne Sprungstellen. Wir führen als Maß für die Dispersion $\delta_{m}$ das Verhältnis $\frac{\Delta \varepsilon}{\varepsilon_{n}}$ ein, wobei $\Delta \varepsilon$ die Differenz zwischen den $\mathrm{DK}$. bei $3 \cdot 10^{2}$ und $10^{8} \mathrm{~Hz}$ und $\varepsilon_{n}$ die DK. bei $3 \cdot 10^{2} \mathrm{~Hz}$ bedeutet. Wir erhalten dann folgende Werte :

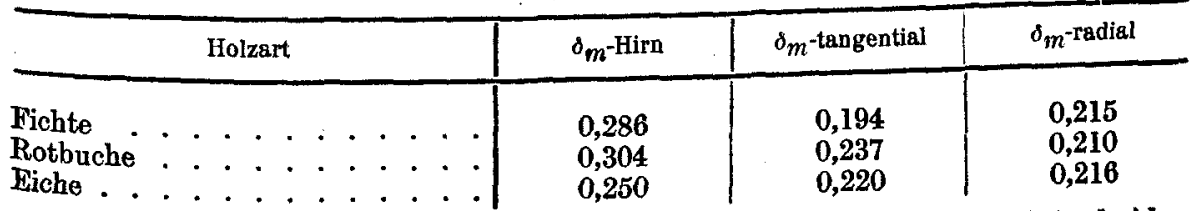

Die größte Dispersion tritt ebenfalls beim Hirnschnitt auf. Die beide anderen Werte sind wenig voneinander verschieden.

\section{Theoretische Betrachtungen}

$\mathrm{DaB}$ ein mechanisch so anisotropes Gebilde wie das Naturholz auch eino dielektrische Anisotropie zeigen würde, erscheint selbstverständlich. 
würde vielleicht auf Grund der Anisotropie bei den mechanischen Eigenschaften noch größere Abweichungen der DK. in verschiedenen Schnittrichtungen erwarten, als sie tatsächlich auftreten. Insbesondere gilt das z. B. für das Eichenholz.

Es entsteht nun die Frage, ob die dielektrische Anisotropie vorzugsweise aus der makroskopischen Struktur des Holzes zu erklären ist oder aus der Feinstruktur der einzelnen Holzfaser, vor allem aus der Anisotropie, bedingt durch die Cellulosekristallite ${ }^{2}$ ) bei einer bestimmten bevorzugten Orientierung. Man könnte also von einer Anisotropie erster Ordnung als Folge der Grobstruktur sprechen und einer solchen zweiter Ordnung, gegeben durch die Feinstruktur.

Die ausgeführten Messungen geben nur die elektrischen Eigenschaften des Holzes als Mischdielektrikum wieder und reichen noch nicht aus, um die Anteile beider Ursachen einer Anisotropie exakt zu trennen. Doch erlauben sie einige Aussagen über ihren EinfluB bei den untersuchten Holzsorten zu machen.

Uber die Ermittlung der DK. eines Mischkörpers aus den Daten der Komponenten besteht ein sehr ausgedehntes Schrifttum. Von den angegebenen Formeln hat sich die von Lichtenecker und Rother ${ }^{3}$ ) angegebene Potenzformel auch praktisch gut bewährt. Sie wird in der von Büchner ${ }^{4}$ ) erweiterten Form angegeben. Es sind $\vartheta_{1}, \vartheta_{2}, \ldots$ die Volumanteile und $\varepsilon_{1}, \varepsilon_{2}, \ldots$ die DK. der einzelnen Komponenten, $k$ eine Konstante, die von der Form und der Verteilung der Komponenten, nicht aber von ihren Volumanteilen abhängt. Dann ergibt sich die DK. des Mischkörpers $\varepsilon$ aus der Gleichung

$$
\varepsilon^{k}=\varepsilon_{1}^{k} \vartheta_{1}+\varepsilon_{2}^{k} \vartheta_{2}+\varepsilon_{3}^{k} \vartheta_{3}+\cdots=\sum_{i=1}^{i=n} \varepsilon_{i}^{k} \vartheta_{i} .
$$

Dabei kann angenommen werden, $\mathrm{da} B$ bei einem Verlustwinkel mit $\operatorname{tg} \delta<0,06$, wie wir es bei den Messungen festgestellt haben, die Formeln für die DK. ohne Korrektur angewandt werden können. Für einen anisotropen Mischkörper, bei dem die einzelnen Komponenten an sich isotrop sind, wie wir es zunächst für das Holz annehmen wollen, sind dann für die drei Achsen die einzelnen $\varepsilon$ und $\vartheta$ gleich und nur $k$ ist verschieden, also:

$$
\begin{aligned}
& \varepsilon_{h}^{k(h)}=\sum_{i=1}^{i=n} \varepsilon_{i}^{k(h) \vartheta_{i}}, \\
& \varepsilon_{i}^{k(t)}=\sum_{i=1}^{i=n} \varepsilon_{i}^{k(t)} \vartheta_{i} \\
& \varepsilon_{r}^{k(r)}=\sum_{i=1}^{i=n} \varepsilon_{i}^{k(r) \vartheta_{i} .}
\end{aligned}
$$

Wir betrachten das $\mathrm{Holz}$ zunächst in erster Annäherung als geschichtetes Dielektrikum aus zwei Komponenten mit den DK. $\varepsilon_{1}$ und $\varepsilon_{2}$. Dabei soll $\varepsilon_{1}$ die DK. der eigentlichen Zellwandungssubstanz als einer der Schichtkomponenten bezeichnen und $\varepsilon_{2}$ die DK. der Luft unter der Annahme, daB im Darrzustande der Einfluß des noch vorhandenen Wassers vernachlässigt werden kann. Dio entsprechenden Volumenanteile sind $\vartheta_{1}$ und $\vartheta_{2}$. Dann ist $\varepsilon_{1}$ wieder dio DK. 
eines zunächst als isotrop angenommenen Mischkörpers zweiter Ordnung, für die sich aus der Gleichung (1) die DK. zu

$$
\varepsilon_{1}^{k(u)}=\sum_{p=1}^{p=m} \varepsilon_{p}^{k(u)} \vartheta_{p}
$$

ergibt. Mit $k(u)$ wird jetzt die Konstante für eine ungeordnete Verteilung bezeichnet.

Für die reine Sohichtung parallel zur Tangentialebene ist bei der Lichten. ecker-Rotherschen Formel $k(h)=k(r)=1$ zu setzen. Die Formel für $\varepsilon_{h}$ bei 2 Mischkomponenten geht dann in diejenige für eine einfache Parallelschaltung über:

$$
\varepsilon_{h}=\varepsilon_{1} \vartheta_{1}+\varepsilon_{2} \vartheta_{2} \quad \varepsilon_{h}=\varepsilon_{r}
$$

Senkrecht zur Schichtebene (Tangentialschnitt) erhält man dann mit $k_{t}=-1$ die Beziehung für die einfache Reihenschaltung

$$
\frac{1}{\varepsilon_{t}}=\frac{\vartheta_{1}}{\varepsilon_{1}}+\frac{\vartheta_{2}}{\varepsilon_{2}}
$$

Das sind die beiden Grenzformeln für einen zweiteiligen Mischkörper. Es fragt sich nun, wieweit man sie annähernd auf das Holz anwenden darf.

Wir gehen vom Fichtenholz aus, das als Nadelholz eine regelmäßige und relativ einfache makroskopische Struktur besitzt. Wir erkennen aus den Kurven der Abb. 2 sofort, daB nicht $\varepsilon_{h}=\varepsilon_{r}$, sondern annähernd $\varepsilon_{t}=\varepsilon_{r}$ ist. Für die Anisotropie ist, wie schon erwähnt, die Faserrichtung maßgebend. Man müßte also an Stelle der Schichtstruktur eine Stäbchenstruktur annehmen, die auch aus dem anatomischen Bau folgt. Die Formel (4) für Parallelschaltung bleibt dann bestehen, nicht aber die Gleichung (5). Es muß bei der Ausgangsformel (2) an Stelle von $k_{t}=-1$ ein anderer noch unbestimmter Faktor eingesetzt werden. Wir wollen aber unter Vorbehalt einer nachträglichen Korrektur zunächst die Grenzformel (5) beibehalten und im wesentlichen eine Schichtung parallel zu den Flächen der Jahresringe annehmen. Dann können wir aus den Gleichungen (4) und (5) unter der Annahme einer bekannten DK. $\varepsilon_{2}$ die Volumenanteile $\vartheta_{1}$ und die DK. $\varepsilon_{1}$ der Zellsubstanz berechnen und mit den aus dem Schrifttum bekannten. Werten vergleichen.

Wir setzen $\vartheta_{2}=\left(1-\vartheta_{1}\right)$ und erhalten dann durch eine einfache Rechnung:

$$
\begin{gathered}
\varepsilon_{1}=\frac{1}{2\left(\varepsilon_{t}-\varepsilon_{2}\right)}\left[\left(\varepsilon_{h} \varepsilon_{t}-\varepsilon_{2}^{2}\right)+\sqrt{\left(\varepsilon_{h} \varepsilon_{t}-\varepsilon_{2}^{2}\right)^{2}-4 \varepsilon_{2} \varepsilon_{t}\left(\varepsilon_{h}-\varepsilon_{2}\right)\left(\varepsilon_{t}-\varepsilon_{2}^{2}\right)}\right] \\
\vartheta_{1}=\frac{\varepsilon_{h}-\varepsilon_{2}}{\varepsilon_{1}-\varepsilon_{2}} .
\end{gathered}
$$

Setzen wir für Fichtenholz im Darrzustand $\varepsilon_{2}=1$ als relative DK. für Luft und für $\varepsilon_{h}$ und $\varepsilon_{t}$ die gemessenen Werte aus dem Diagramm Abb. 2 ein, so erhält man für $\varepsilon_{1}$ und $\vartheta_{1}$ die in gleicher Abbildung angegebenen Werte. Im Mittel erhält man für $\vartheta_{1}$ den Wert 0,58 . Bei $f=300$ ist $\varepsilon_{1}=4,40$, während man auf Grund der Messungen von Büchner ${ }^{5}$ ) für die DK. der Faser des Hadernpapieres und der Natron- und Sulfitcellulose im Mittel etwa 5,6 erhält. Nach den Messungen von Kröner ist anzunehmen, daB sich die DK, des Lignins 
als des zweitwichtigsten Bestandteils der Zellwandung nur wenig von der DK. der Cellulose unterscheidet. Wir nehmen diese Zahl als geltend für den Stoff der Zellwandungen an. Wir betrachten weiter die Schichten des festen Körpers als annähernd isotrope Mischkörper aus Luft und Zellsubstanz und berechnen daraus den Anteil der Komponenten. Für Mischkörper mit ungeordneter Verteilung ist nach Lichtenecker $k=0$ zu setzen. Die Ableitung aus den Ausgangsgleichungen ergibt dann die bekannte logarithmische Mischformel

$$
\log \varepsilon_{1}=\vartheta_{3} \log \varepsilon_{2}+\vartheta_{4} \log \varepsilon f
$$

$\left(\vartheta_{3}, \vartheta_{4}-\right.$ die Volumenanteile von Luft und Zellsubstanz, $\varepsilon_{f} \cdots$ DK. der Zellsubstanz).

Wir setzen die Werte $\varepsilon_{2}=1 ; \varepsilon_{1}=4,40 ; \varepsilon_{f}=5,6$ ein und berechnen daraus $\vartheta_{4}=0,85$. Das ist der Anteil des Zellkörpers am Körper der einzelnen Schicht. Das Gesamtvolumen des Zellkörpers, bezogen auf den ganzen Körper, ist dann

$$
\vartheta_{1}^{\prime}=0,58 \cdot 0,85=0,49 \text {. }
$$

Aus dem Schrifttum ${ }^{6}$ ) kann man den Volumenanteil der Zellsubstanz für Fichtenholz zu 0,35 bis 0,42 entnehmen, wobei 0,42 dem Höchstwert entspricht. Der berechnete Wert liegt über dem Höchstwert.

Wir machen nun eine andere Annahme und gehen von der Feinstruktur aus. Die Cellulosekristallite sind besonders bei dem Nadelholz, wie es die Röntgendiagramme zeigen, vorzugsweise in Richtung der Stammachse orientiert. Wir versuchen nun, die Anisotropie aus der Anordnung von Elementen abzuleiten, die vorzugsweise nach der $h$.Achse ausgerichtet sind und in der dazu senkrechten Ebene, also im Querschnitt, eine annähernd gleichmäßigeVerteilung zeigen. Als zweite Komponente nehmen wir wieder Luft an. Wir nehmen zunächst den ganzen Volumenanteil des Zellgewebes von 0,4 für die Cellulose in Anspruch. Es wird also für die amorphen und unpolaren festen Bestandteile (Lignin usw.) die gleiche DK. angenommen wie für Cellulose. Wir werden sehen, daß wir dadurch die zweite Annahme noch begünstigen. Für die Ermittlung der DK. der Fasern bei einer Feldrichtung längs der Achse, also im Hirnschnitt, ist dann die Gleichung (4) für reine Parallelschaltung anzuwenden. Für den Tangentialschnitt kommt die bekannte Rayleighsche Formel für eine Verteilung annähernd zylindrischer Elemente mit paralleler Achse in einem sonst homogenen Medium, also hier in Luft, in Frage. Für die DK. der reinen Faserelemente bei einer Feldrichtung senkrecht zur Längsachse lautet diese Formel:

$$
\varepsilon_{f t}=\frac{\varepsilon_{2}\left(\vartheta_{1}+\frac{\varepsilon_{t}-\varepsilon_{2}}{\varepsilon_{t}+\varepsilon_{2}}\right)}{\vartheta_{1}-\frac{\varepsilon_{t}-\varepsilon_{2}}{\varepsilon_{t}+\varepsilon_{2}}}
$$

Nach Einsetzen von $\varepsilon_{2}=1$ und des gemessenen Wertes für $\varepsilon_{t}=1,92$ bei $\vartheta_{1}=0,4$ erhält man aus den beiden Gleichungen

$$
\begin{gathered}
\varepsilon_{f h}=9,4, \\
\varepsilon_{f t}=8,3 .
\end{gathered}
$$

Die Anisotropie der einzelnen Faser wäre also nur wenig ausgeprägt. Vor allem erhält man aber für die DK. Werte, die sogar im Mittel weit über dem von Büchner für die Faser gefundenen Mittelwert von 5,6 liegen. Nimmt man 
kleinere Volumenanteile für den festen Körper an als 0,4 , so werden die berechneten DK.-Werte noch größer. Es muß daher als wahrscheinlich angenommen werden, daß durch den großen Volumenanteil der Luft die durch die Feinstruktur bedingte Anisotropie nur wenig in Erscheinung tritt und die makroskopische Struktur maßgebend ist. Der zu große vorhin bei der Annahme einer Schichtstrulktur berechnete Volumenanteil der festen Substanz ist aus der natürlichen Ungleichmäßigkeit des Holzes als Stoff zu erklären. Jedenfalls sind diese Abweichungen von den wahrscheinlichen Werten wesentlich kleiner als bei der zuletzt gemachten Annahme, die die Anisotropie aus der Feinstruktur abzuleiten versucht. Das gilt zunächst für das Fichtenholz als Nadelholz mit relativ regelmäßigem makroskopischen Aufbau.

Bei Laubhölzern mit ihrer wesentlich verwickelteren Struktur ist im allgemeinen eine größere Abweichung von dem geschichteten Schema zu erwarten. Bei einer der gemessenen Proben von Rotbuchenholz, für die die Werte in Abb. 3 angegeben sind, ist die Anisotropie geringer als bei Fichte. Der berechnete Wert für den Volumenanteil des festen Stoffes von 0,8 weicht wesentlich mehr von den für Buche bekannten Werten von etwa 0,5 ab, als wir es bei Fichte gefunden hatten. Eine Versuchsreihe mit einer anderen Probe von Buchenholz ergab bessere Úbereinstimmung. Es wurden hier bei einer Frequenz von $500 \mathrm{~Hz}$ die Werte $\varepsilon_{h}=3,23$ und $\varepsilon_{t}=2,0$ gemessen. Es wird daraus $\varepsilon_{1}=4,70$ und $\vartheta_{1}=0,54$ berechnet.

Das Eichenholz zeigt eine noch geringere Anisotropie, so daß das reine Schichtschema zur Erklärung der dielektrischen Eigenschaften nicht in Frage kommt. Trotzdem widersprechen die Ergebnisse für die Laubhölzer nicht der allgemeinen Anschauung, daß die makroskopische Struktur die dielektrische Anisotropie in erster Linie verursacht. Nur ist der Einfluß wegen des komplizierteren Aufbaus schwerer zu verfolgen.

Die Dispersion der DK. kann bei einem Mischdielektrikum entweder aus den molekularen Dipoleigenschaften der Komponenten (Debyesche Dipoltheorie) oder aus der Inhomogenität des Körpers selbst (Wagnersche Theorie) erklärt werden. Die Entscheidung darüber, welche Auffassung für unseren Fall als wahrscheinlich angenommen werden kann, erfordert eingehende Untersuchung über die Abhängigkeit der Verluste von der Frequenz und fältt aus dem Rahmen der vorliegenden Arbeit. Soviel aber soll hier gesagt werden, daß nach den von uns durchgeführten Untersuchungen, über die an anderer Stelle berichtet werden soll, die polaren Eigenschaften der Cellulose in erster Linie für die Erklärung der Dispersion in Frage kommen ${ }^{7}$ ). Hier interessiert uns die Abhängigkeit der Dispersion von der Feldrichtung. Wie es aus den auf $S_{4} 98$ gegebenen Daten zu ersehen ist, hat man bei einer Feldrichtung senkrecht zum Hirnschnitt eine größere Dispersion als bei den anderen Richtungen. Die Größe der Dispersion steht also mit dem absoluten Wert der DK. bei der gleichen Feldrichtung in unmittelbarem Zusammenhang.

Bei Annahme des geschichteten Schemas läßt sich das Verhalten der Dis persion in der $h$ - und $t$-Achse einfach aus den Gleichungen (4) und (5) ableiten. Wir setzen für die absolute Dispersion ( $f=$ Frequenz)

$$
\delta_{h}=\frac{d \varepsilon_{h}}{d f} ; \quad \dot{\delta}_{t}=\frac{d \varepsilon_{t}}{d f}
$$


108 K. Kröner und L. Pungs: Zur elektrischen Anisotropie des Naturholzes uaw.

Das Verhältnis der Dispersionen für die beiden Achsen ergibt sich zu

$$
v=\frac{\delta_{h}}{\delta_{t}}=\frac{d \varepsilon_{h}}{d \varepsilon_{t}}
$$

Aus den Gleichungen erhält man bei $\varepsilon_{2}=1$

$$
\begin{gathered}
d \varepsilon_{h}=\vartheta_{1} d \varepsilon_{1}^{\prime \prime}, \\
d \varepsilon_{t}=\frac{1}{\vartheta_{1}+\vartheta_{2} \varepsilon_{1}} \cdot\left(1-\frac{\varepsilon_{1} \vartheta_{2}}{\vartheta_{1}+\vartheta_{2} \varepsilon_{1}}\right) d \varepsilon_{1} .
\end{gathered}
$$

Für Fichtenholz bei $f=10^{4}$ erhält man $v=5,3$. Die Kurven der Abb. 2 ergeben annähernd $v=4,0$. Dabei sind die Einlagerungen der Luft in den Schichten nicht berücksichtigt. Es muß als sehr wahrscheinlich angenommen werden, daß die Veränderungen der Dispersion mit der Feldrichtung ebenfalls durch die makroskopische Struktur bedingt sind.

\section{Zusammenfassung}

Die dielektrische Anisotropie von Holl (Fichte, Buche und Eiche) im Darrzustande ist durch Messungen der Dielektrizitätskonstante im Frequenzbereich von $3 \cdot 10^{2}$ bis $10^{9} \mathrm{~Hz}$ untersucht. Es ist gefunden worden, daß die Anisotropie vor allem durch die makroskopische Struktur von Holz als eines Mischkörpers aus Zellsubstanz und Luft bedingt ist. - Eine anomale Dispersion der Dielektrizitätskonstante ist festgestellt worden. Die Änderungen der Dispersion bei verschiedenen Richtungen des elektrischen Feldes sind ebenfalls durch die Mischstruktur erklärt worden.

\section{Literatur}

) Helmhoitz, Vorlesungen über theoretische Physik 1902, Bd. II, S. 103.

9) Freudenberg, Zocher u. Dürr, Ber. chem. Ges. 62, S. 1814.

- Lich tenecker u. Rother, Phys. Zeitschr. 32, 255, 1931.

4) Wissenschaftl. Veröffentl. des Siemens-Konzerns 18, Heft 2, 84, 1939.

- Btichner, Wiss. Veröffentl. des Siemens-Konzerns 18, Heft 2, 84, 1939.

9) Kollmann, Technologie des Holzes 1936, S. 39.

7) Kröner, Dissertation Braunschweig 1943. 